\title{
A Novel Method for Frequency Estimation Considering Instrument Transient Effect
}

\author{
Mohsen Tajdinian, Mehdi Zareian Jahromi, Mojtaba Jalalpour \\ Dept. of Electrical Engineering, Amirkabir University of Technology \\ No. 424, Hafez Avenue, Tehran 1591634311, Iran \\ e-mail: jalalpur@aut.ac.ir
}

\begin{tabular}{l} 
Article Info \\
\hline Article history: \\
Received Nov 12, 2014 \\
Revised Jan 10, 2015 \\
Accepted Jan 26, 2015 \\
\hline Keyword: \\
CCVT transient \\
Frequency estimation \\
Least square technique \\
Phasor estimation
\end{tabular}

\begin{abstract}
Large disturbances in power systems cause deviation in the frequency from the nominal value. Since the frequency is an important factor in the electrical network parameter measurements, it can cause malfunction of the protection system. In addition, Because of decaying DC and oscillatory components that introduced by CCVT in response of voltage variation during the fault occurrence, cause changes in the value of received voltage of primary side of CCVT. An improved least square method for estimating frequency is presented in this paper. In order to reduce the effect of this transient component, phasor estimation method has been improved by using the least square technique and utilizing knowledge of CCVT design. The capability of the proposed method was verified by several case studies generating signals in PSCAD/EMTDC. The results show the accuracy, speed and capability of the proposed method.
\end{abstract}

Copyright (C) 2015 Institute of Advanced Engineering and Science. All rights reserved.

\section{Corresponding Author:}

Mojtaba Jalalpour,

Dept. of Electrical Engineering, Amirkabir University of Technology

No. 424, Hafez Avenue, Tehran 1591634311, Iran

Email: jalalpur@aut.ac.ir

\section{INTRODUCTION}

The most important devices used for power system security, are protective relays. in the presence of fault, these devices must identify the faulty section or component correctly and quickly and isolate it so that other sections of the network have a minimum impact. This simple and basic requirement is hard to achieve in presence of transient components due to fault, which is present in voltage and current signals received by the protective device. The coupling capacitor voltage transformer (CCVT) is one of the main devices whose transient response impacts on performance of a distance relay. Although the voltage output of CCVT at steady-state condition is accurate, its behavior during transient causes inaccuracy in phasor calculation. Moreover Frequency is a significant operating parameter of a power system. Precise monitoring of the frequency of a power system is important to optimal operation, especially a real-time precise estimation of the main frequency is necessary for electrical parameter measurements. This is because numerous numerical methods for electrical parameter measurements are sensitive to frequency fluctuations.

Several methods for estimating frequency have been mentioned in the technical literature [1-18]. Zero-crossing methods are the most widely used methods for estimating frequency [1, 2]. However, their performance is Impressive to switching-type transients. The Kalman filter has also been utilized for frequency estimation in power system [3,4], but it is highly affected by the initial conditions and its performance is not robust due to deviations in the internal parameters of the model. A three-phase, phaselocked loop (PLL) prepares quick and robust frequency estimation for balanced three-phase networks and has been widely used for estimating frequency [5-7]. Nevertheless, its performance is prone to error in unbalanced conditions. different approaches, consist of least square methods [10, 11], DFTs [14-16], 
Newton-based algorithms [12], and the maximum likelihood approach [17] ,adaptive neural networks (ANN) $[8,9]$, complex Prony analysis [13, 18] have also been utilized for frequency estimation of power system signals.

A new dynamic frequency estimation method is suggested in this paper. This method which is based on least square technique uses the CCVT transient information in the voltage waveform which is displayed during transient condition. Some case studies such as signal test and also significant cases for fault in transmission line are presented. Signal is generated by PSCAD/EMTDC software. With CCVT information and proposed algorithm, the results show that speed of convergence and accuracy has been improved in the frequency.

\section{BACKGROUND}

\subsection{Effective Factors on the Transient Response of a CCVT}

The most significant factors that impact the CCVT transients response are the power factor and magnitude of secondary burden, the incidence angle of fault, voltage change magnitude and Ferro resonance Suppression Circuit (FSC) of CCVT [21], [22].

\subsubsection{Burden}

Most CCVT models give proper performance during the transient state for burdens lower than the nominal burden. Whenever, the power factor decreases, CCVT transient response becomes worse. Parallel RL circuits' burdens for the same volt-ampere and power factor give the worse transient response than Series RL [21]. The inductive burdens found in electromechanical relays cause more CCVT transients state than the resistive burdens found in digital and numerical relays [23].

\subsubsection{Fault Incidence Angle}

Incidence angle of the fault causes changes in CCVT transient behavior. [23]. For a voltage peak transient, the resulting similar is an under damped system that will cause a transient voltage that oscillates at a frequency larger than the power frequency. A voltage zero transients cause an overshoot voltage that damps to zero in a few cycles. In this case, the subsidence voltage will result in a reduced voltage magnitude and shifted phase angle [24]. Faults at zero crossing of the primary voltage cause significant transient errors that result in the operation of covered protective relays [25].

\subsubsection{FSC Type}

There are two types of FCSs, 'passive' type, which do not store energy and 'active' type, which stores energy [20], [21]. Active ferroresonance suppression circuits (AFSC) have parallel connection of iron core inductors and capacitors, which is adjusted to the power frequency. These circuits consistently connected on CCVT secondary side [23], [26]. The AFSC behaves like a band-pass filter and causes additional time delay in the CCVT secondary output. The energy storage components in the AFSC participate to the intensity of the CCVT transients [23]. Passive ferroresonance-suppression circuits (PFSC) consist of a consistently connected loading resistor and a saturable inductor. The saturable inductor is designed to saturate at about $150 \%$ of rated voltage to hold up a sustained ferroresonance condition [23]. These FSCs do not change transient response of CCVT unless an overvoltage occurs [26].

\subsubsection{Magnitude of Voltage Change}

The fault voltage magnitude level is the significant factor that affects the intensity of CCVT transients. In the lower voltage level, the transient response of Secondary voltage wave of CCVT is more heterogeneous in during the fault. The voltage change magnitude depends to source to line impedance ratio (SIR) value, fault location, and fault resistance [23]. A higher SIR causes into a larger magnitude voltage change at the relay location on the onset of a fault [22], which can result in higher overreach difficulties.

\section{2 . Equivalent Circuit of a CCVT}

A general coupling capacitor voltage transformer (CCVT) includes a capacitive voltage divider, tuning reactor, step-down transformer and Ferro resonance suppression circuit. During fault conditions, because of the voltage drops, there is no threat of over passing the knee-point of the magnetizing characteristic of the step-down transformer, so a CCVT can be expressed by the equivalent linear circuit as illustrated in Figure 1. In this paper the CCVT model shown in Figure 1. The linear circuit of Figure 1 can be further simplified as shown in Figure 2. The parameters in the circuit of Figure 2 are: 


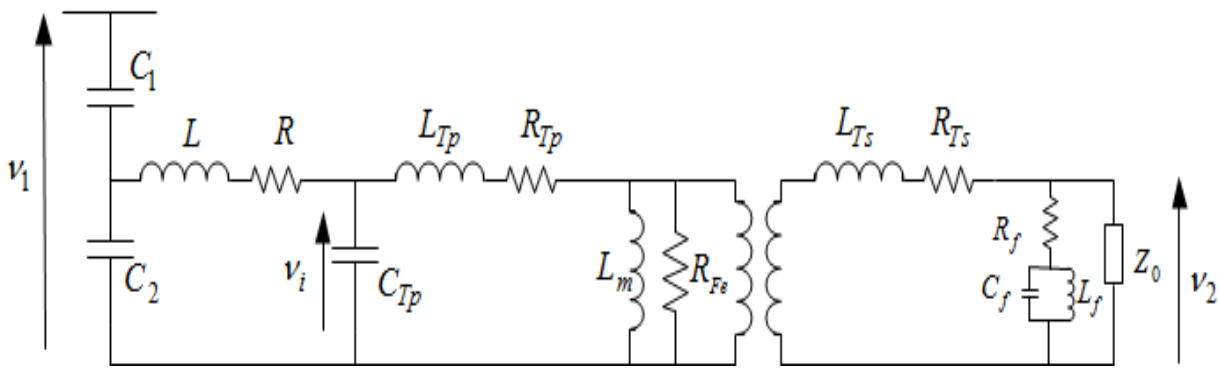

Figure 1. Equivalent circuit diagram of a CCVT

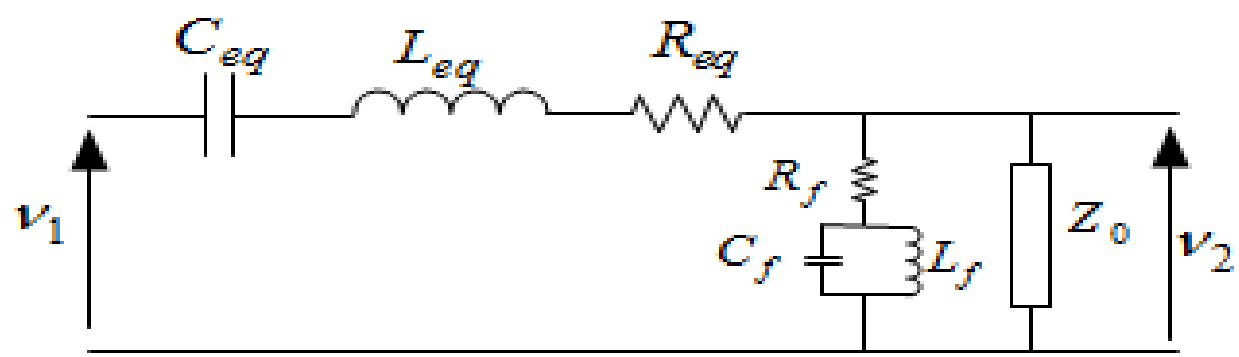

Figure 2. Simplified model of a CCVT from Figure 1

$C_{e q}$ is sum of the stack capacitances, $L_{e q}$ and $R_{e q}$ are equivalent inductance and resistance respectively, of the tuning reactor and the Step down transformer, $Z_{0}$ is burden impedance, $f$ is subscript for parameters of the anti-resonance circuit.

As mentioned in [19], the transfer function of a CCVT derived for the model of Figure 2 is:

$$
G(s)=\frac{K_{C C V T}\left(s-z_{1}\right)\left(s-z_{1}^{*}\right)\left(s-z_{2}\right)\left(s-z_{3}\right)}{\left(s-p_{1}\right)\left(s-p_{1}^{*}\right)\left(s-p_{2}\right)\left(s-p_{2}^{*}\right)\left(s-p_{3}\right)}
$$

Where $z_{i} \mathrm{~S}$ is zeros of CCVT transfer function and $p_{i}$ s are poles of CCVT transfer function and:

$$
\begin{aligned}
& p_{1}=-\alpha_{1}+j \omega_{1} \\
& p_{2}=-\alpha_{2}+j \omega_{2} \\
& p_{3}=-\alpha_{3}
\end{aligned}
$$

Where $p_{1}$ is low frequency pole, $p_{2}$ is high frequency pole and $p_{3}$ is dc pole.

\section{PROPOSED METHOD}

In order to investigate the behavior of CCVT, the CCVT input signal has been considered during fault occurrence has been considered as following:

$$
v(t)=B e^{-\frac{t}{\tau}}+A_{1} \sin \left(\omega t+\varphi_{i}\right)+\sum_{i=2}^{N} A_{i} \sin \left(i \omega_{0} t+\varphi_{i}\right)
$$

Where $B$ is the amplitude and $\tau$ is the time constant of decaying dc signal, $A_{i}$ s are harmonic components including signal fundamental component, $\omega_{0}$ is fundamental angular frequency which $\omega_{0}=2 \pi f_{0}$ 
and $f_{0}$ is the main frequency of the system. The exponential DC term of signal is created when fault occurs in the network and the other sinusoidal expressions are second and higher order harmonic of signal of voltage.

As has been mentioned in [19], the CCVT amplitude frequency response is such that DC component and high-frequency components eliminates and also strengthens or weakens frequency of around the power frequency. The fifth-order transfer function can be used to represent CCVT behavior [19]. According to this model, the output signal from CCVT is generally to be considered as follows:

$$
\begin{aligned}
\hat{v}(t) & =V_{r} \cos (\omega t)+V_{i} \sin (\omega t) \\
& +V_{r 1} e^{-\alpha_{1} t} \cos \left(\omega_{1} t\right)+V_{i 1} e^{-\alpha_{1} t} \sin \left(\omega_{1} t\right) \\
& +V_{r 2} e^{-\alpha_{2} t} \cos \left(\omega_{2} t\right)+V_{i 2} e^{-\alpha_{2} t} \sin \left(\omega_{2} t\right)+V_{d c} e^{-\alpha_{d c} t}
\end{aligned}
$$

Where $V_{r}$ and $V_{i}$ are real and imaginary parts of voltage phasor of fundamental components, $\alpha_{1}$ and $\omega_{1}$ and $\alpha_{2}$ and $\omega_{2}$ are real and imaginary parts and $\alpha_{d c}$ is dc component pole of the transfer function of CCVT. Utilizing Taylor series, $\sin (\omega t), \cos (\omega t)$ can be expanded as below :

$$
\begin{aligned}
& \sin (\omega t) \approx \sin \left(\omega_{0} t\right)+t \Delta \omega \cos \left(\omega_{0} \mathrm{t}\right)-\frac{t^{2}}{2} \Delta \omega^{2} \sin \left(\omega_{0} t\right) \\
& \cos (\omega t) \approx \cos \left(\omega_{0} t\right)-t \Delta \omega \sin \left(\omega_{0} \mathrm{t}\right)-\frac{t^{2}}{2} \Delta \omega^{2} \cos \left(\omega_{0} t\right)
\end{aligned}
$$
follow:

By substituting Taylor series expansion in (6), the output signal of the CCVT can be represented as

$$
\begin{aligned}
\hat{v}(t)= & V_{r} \cos (\omega t)+V_{i} \sin (\omega t)+V_{r}^{\prime} t \cos (\omega t) \\
& +V_{i}^{\prime} t \sin (\omega t)+V_{r}^{\prime \prime} t^{2} \cos (\omega t)+V_{i}^{\prime \prime} t^{2} \sin (\omega t) \\
& +V_{r 1} e^{-\alpha_{1} t} \cos \left(\omega_{1} t\right)+V_{i 1} e^{-\alpha_{1} t} \sin \left(\omega_{1} t\right) \\
& +V_{r 2} e^{-\alpha_{2} t} \cos \left(\omega_{2} t\right)+V_{i 2} e^{-\alpha_{2} t} \sin \left(\omega_{2} t\right)+V_{d c} e^{-\alpha_{d c} t}
\end{aligned}
$$

\subsection{Calculation of Phasor}

Damping oscillatory components and dc decaying component of the voltage signal which is created during the fault occurrence, cause the unsuccessful estimation with basic least square method. in order to overcome this problem, it must be considered that the components of transient response CCVT has been extracted from the equivalent circuit section 2.1, phasor estimation can be improved by the least squares method. According to the equation (9) can be written:

$$
\hat{V}[m \times 1]=D[m \times 11] \times E[11 \times 1]
$$

where:

$$
\begin{aligned}
& \hat{V}=\left[\begin{array}{llllll}
\hat{v}\left(t_{1}\right) & \hat{v}\left(t_{2}\right) & \hat{v}\left(t_{3}\right) & \hat{v}\left(t_{4}\right) & \cdots & \hat{v}\left(t_{m}\right)
\end{array}\right]^{T} \\
& {[D]_{m \times 11}=\left[\begin{array}{lllll}
{\left[d_{1}\right]_{m \times 3}} & {\left[d_{2}\right]_{m \times 3}} & {\left[d_{3}\right]_{m \times 1}} & {\left[d_{4}\right]_{m \times 2}} & {\left[d_{5}\right]_{m \times 2}}
\end{array}\right]} \\
& E=\left[\begin{array}{lllllllllll}
V_{r} & V_{r}^{\prime} & V_{r}^{\prime \prime} & V_{i} & V_{i}^{\prime} & V_{i}^{\prime \prime} & V_{d c} & V_{r 1} & V_{i 1} & V_{r 2} & V_{i 2}
\end{array}\right]^{T}
\end{aligned}
$$

where in (12) 
$d_{1}=\left[\begin{array}{ccc}\cos \left(\omega t_{1}\right) & t_{1} \cos \left(\omega t_{1}\right) & t_{1}{ }^{2} \cos \left(\omega t_{1}\right) \\ \cos \left(\omega t_{2}\right) & t_{2} \cos \left(\omega t_{2}\right) & t_{2}{ }^{2} \cos \left(\omega t_{2}\right) \\ \cos \left(\omega t_{3}\right) & t_{3} \cos \left(\omega t_{3}\right) & t_{3}{ }^{2} \cos \left(\omega t_{3}\right) \\ \vdots & \vdots & \vdots \\ \cos \left(\omega t_{m}\right) & t_{m} \cos \left(\omega t_{m}\right) & t_{m}{ }^{2} \cos \left(\omega t_{m}\right)\end{array}\right]$

$d_{2}=\left[\begin{array}{ccc}\sin \left(\omega t_{1}\right) & t_{1} \sin \left(\omega t_{1}\right) & t_{1}^{2} \sin \left(\omega t_{1}\right) \\ \sin \left(\omega t_{2}\right) & t_{2} \sin \left(\omega t_{2}\right) & t_{2}^{2} \sin \left(\omega t_{2}\right) \\ \sin \left(\omega t_{3}\right) & t_{3} \sin \left(\omega t_{3}\right) & t_{3}^{2} \sin \left(\omega t_{3}\right) \\ \vdots & \vdots & \vdots \\ \sin \left(\omega t_{m}\right) & t_{m} \sin \left(\omega t_{m}\right) & t_{m}{ }^{2} \sin \left(\omega t_{m}\right)\end{array}\right]$

$d_{3}=\left[\begin{array}{c}e^{-\alpha_{\mu_{1}}} \\ e^{-\alpha_{\mu_{2}} t_{2}} \\ e^{-\alpha_{\mu_{3}} t_{3}} \\ \vdots \\ e^{-\alpha_{\mu_{m}} t_{m}}\end{array}\right]$

$d_{4}=\left[\begin{array}{cc}e^{-\alpha_{1} t_{1}} \cos \left(\omega_{1} t_{1}\right) & e^{-\alpha_{t_{1}}} \sin \left(\omega_{1} t_{1}\right) \\ e^{-\alpha_{1} t_{2}} \cos \left(\omega_{1} t_{2}\right) & e^{-\alpha_{1} t_{2}} \sin \left(\omega_{1} t_{2}\right) \\ e^{-\alpha_{1} t_{3}} \cos \left(\omega_{1} t_{3}\right) & e^{-\alpha_{t_{3} t_{3}}} \sin \left(\omega_{1} t_{3}\right) \\ \vdots & \vdots \\ e^{-\alpha_{1} t_{m}} \cos \left(\omega_{1} t_{m}\right) & e^{-\alpha_{1} t_{m}} \sin \left(\omega_{1} t_{m}\right)\end{array}\right]$

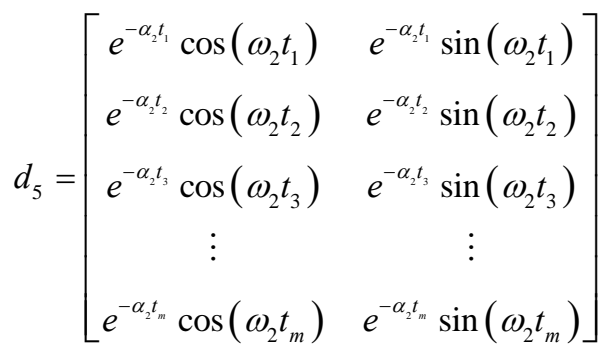

In the equation (10) $\widehat{V}$ and $E$ are value of known and unknown factors respectively and $D$ is the matrix of known values. The equation (19) is obtained by using the method of least squares (LS):

$$
E=\left(D^{T} D\right)^{-1} D^{T} X
$$

By calculating this matrix, real and imaginary values of the signals will be obtained.

$$
\bar{V}=V_{r}+j V_{i}
$$




\subsection{Frequency Estimation}

In practice, three phase fault doesn't occur constantly. Actually the continuity of single phase faults which are much more common causes phase-phase and three phase faults and also voltage unbalances. Solving this problem, positive sequence of the voltage signals can be used [27]. In other words, voltage positive sequence is always available under balanced and unbalanced conditions.

Referring to above reasonable assumptions, the positive sequence of voltage signal can be considered into two orthogonal components as follows:

$$
\begin{aligned}
& V_{r}(t)=V_{m} \cos (\omega t+\theta) \\
& V_{i}(t)=V_{m} \sin (\omega t+\theta)
\end{aligned}
$$
is obtained:

By differentiation of equations (21) and (22) and the placement in each other, the following equation

$$
f=\frac{V_{r e}(t) V_{i m}^{\prime}(t)-V_{i m}(t) V_{r e}^{\prime}(t)}{2 \pi\left(V_{r e}^{2}(t)+V_{i m}^{2}(t)\right)}
$$

Also for created calculation error by approximate differentiation, it can be written:

$$
f_{\text {error }}=\frac{2 \pi^{2} f_{0}^{3} \Delta T^{2}}{3}
$$

As a result, the frequency can be calculated as follows:

$$
f_{\text {estimated }}=f+f_{\text {error }}
$$

\subsection{Algorithm Implementation and Computation Process}

The proposed algorithm is described in previous section. Computing process of the algorithm is shown in Figure (3):

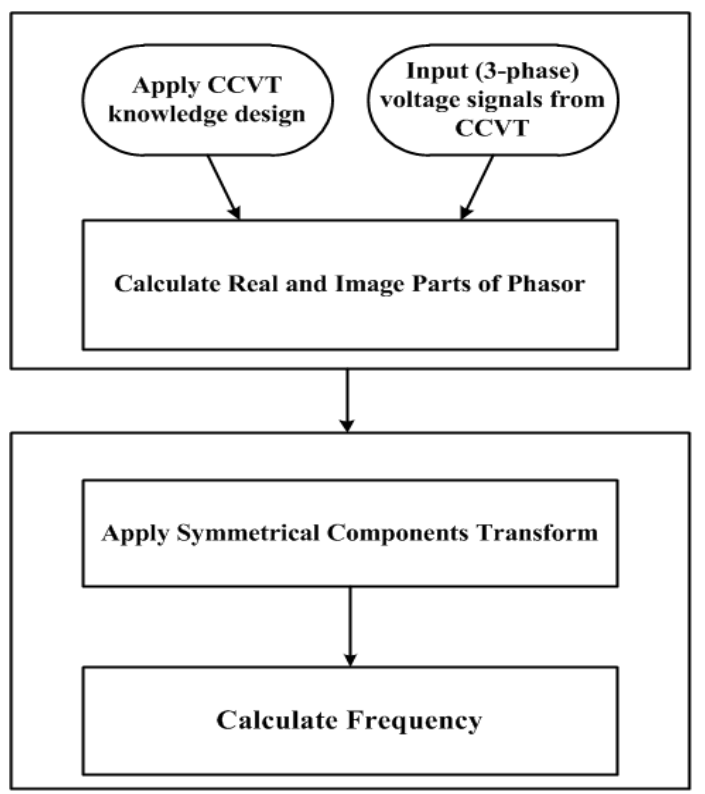

Figure 3. Stages of phasor and frequency estimation 


\section{CASE STUDY}

Dynamic conditions are analyzed using the Electromagnetic Transients Program (PSCAD/EMTDC) to simulate the simple system. As mentioned in section 3; the frequency response of the CCVT, contains attenuated high order harmonic components. Nevertheless, to prevent the presence of high order harmonic components, the second order butterworth lowpass filter with $360 \mathrm{~Hz}$ cutoff frequecny has been used. Also the $5 \mathrm{KHz}$ sampling frequency has been selected. In order to evaluvate the performance of the proposed method (PM), the results of the PM have been compared with Nonlinear Least Squares (NLS) [9], Recursive Discrete Fourier Transform (RDFT) [14] and Adaptive Method (AM) [11]. As shown in figure (4), several dynamic conditions have been selected: double phase-to-ground faults, three phase-to-ground and single phase-to-ground faults in different locations.

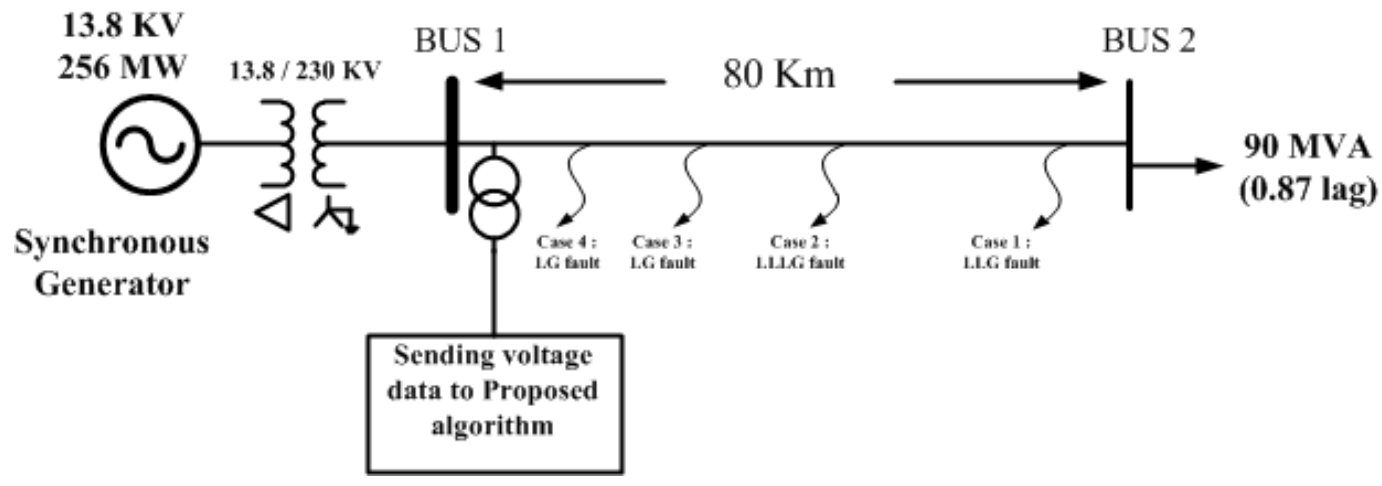

Figure 4. Four kinds of dynamic conditions: double phase-to-ground faults, three phase-to-ground and single phase-to-ground faults in different locations

\subsection{Case1}

Figure (5) shows the time response of the phase A voltage signal for LLG fault condition with a fault incidence angle of $0^{\circ}$ degrees. A magnitude of voltage change of $24 \%(=100 \%-76 \%)$ of the prefault amplitude was observed at this location of the power system selected.

Figures 6 illustrates the frequency of the voltage phasor has been estimated using the (PM). As can be seen in figure (6), proposed approach causes estimated frequency converge to original value after 1 cycle. The results show that AM, RDFT and NLS methods have more sensitivity to Transient component produced by CCVT in voltage signal. That's the reason that frequency converge to original value afte 3 to 5 cycles when CCVT impact is not considered.

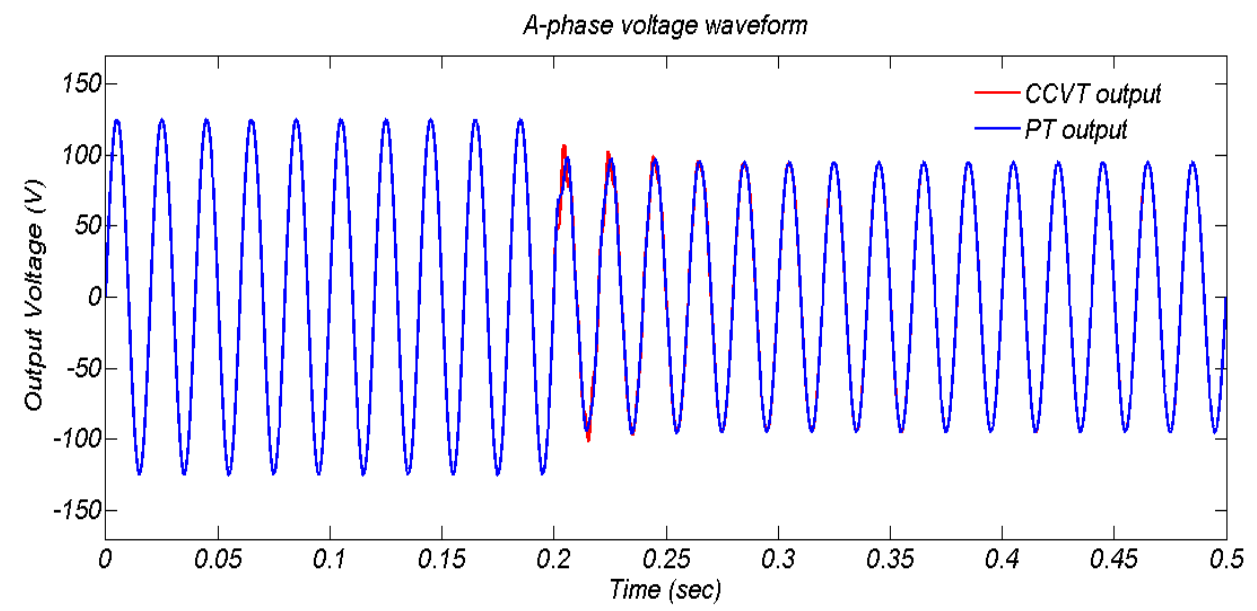

Figure 5. A-phase voltage waveform of the low resistance LLG fault stages of phasor and frequency estimation 


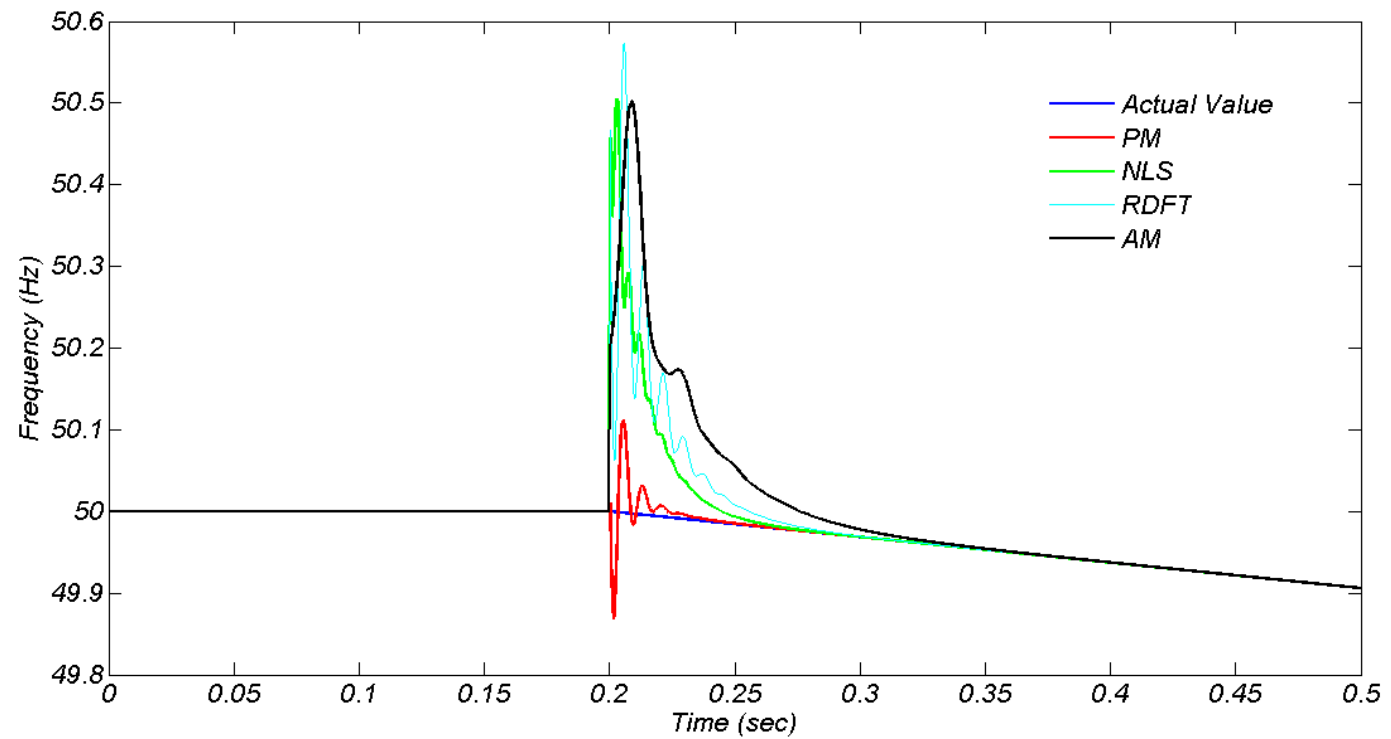

Figure 6. CCVT impact on Frequency during first cycles after fault occurrence

\subsection{Case2}

Figure (7) shows the time response of the phase A voltage signal for LLLG fault condition with a fault incidence angle of $90^{\circ}$ degrees. A magnitude of voltage change of $56 \%$ (= $\left.100 \%-44 \%\right)$ of the prefault amplitude was observed at this location of the power system selected.

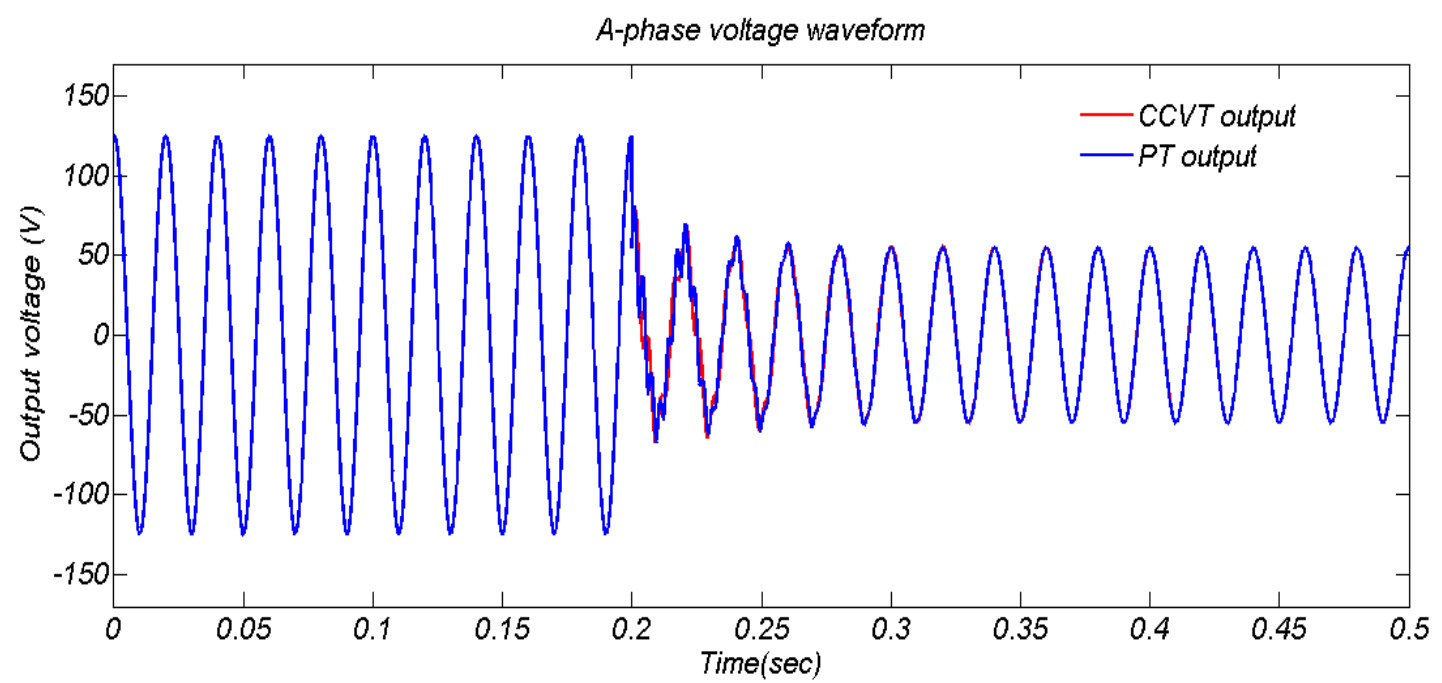

Figure 7. A-phase voltage waveform of the low resistance LLLG fault

Figure (8) illustrates the frequency of the voltage phasor which has been estimated using the proposed method. As can be seen in figure 8, although the stresses produced in voltage signal have been increased, the proposed approach estimated frequency converges to original value after 1 cycle. As shown in figure 8, without consideration of CCVT impact, it takes 3 to 6 cycles for frequency to converge to original value. 


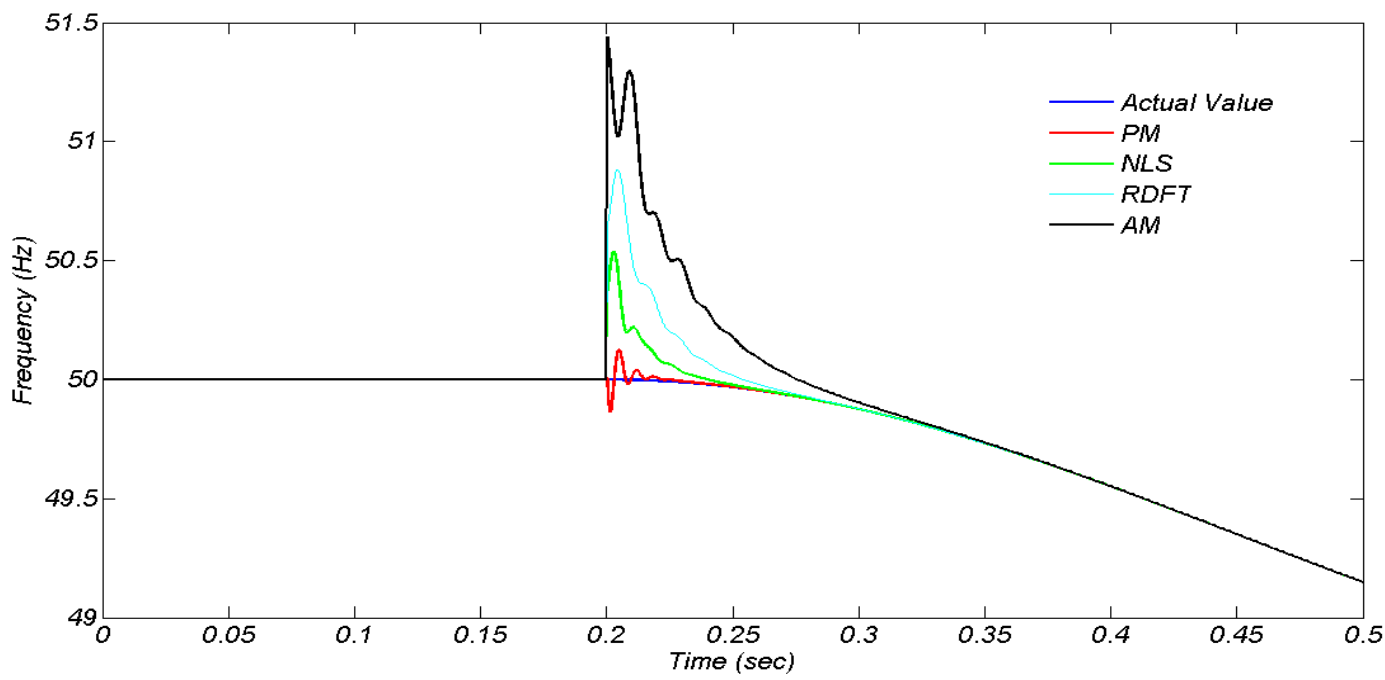

Figure 8. Impact on Frequency during first cycles after fault occurrence

\subsection{Case3}

Figure (9) shows the time response of the phase A voltage signal for LG fault condition with a fault incidence angle of $30^{\circ}$ degrees. A magnitude of voltage change of $64 \%(=100 \%-36 \%)$ of the pre-fault amplitude was observed at this location of the power system selected.

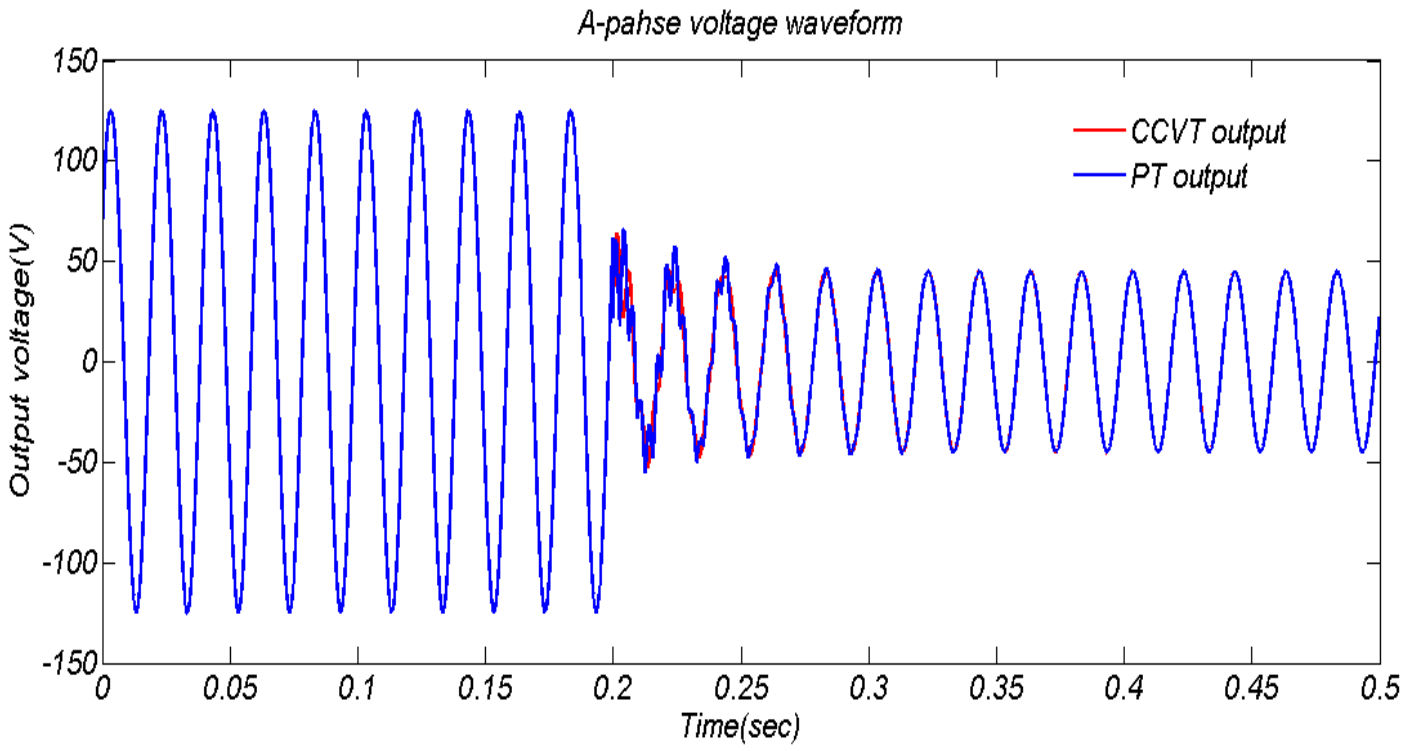

Figure 9. A-phase voltage waveform of the low resistance LG fault

Figure (10) illustrates the frequency of the voltage phasor estimated using the proposed method. As it is shown figure 10, when fault location approaches to the generator bus, stresses in voltage waveform increse. Nevertheless estimated frequency of the proposed method converges to original value after 1 cycle with minimum error. As the results of the proposed method show, consideration of CCVT impact results high precision in transient period in comparison with the other methods. 


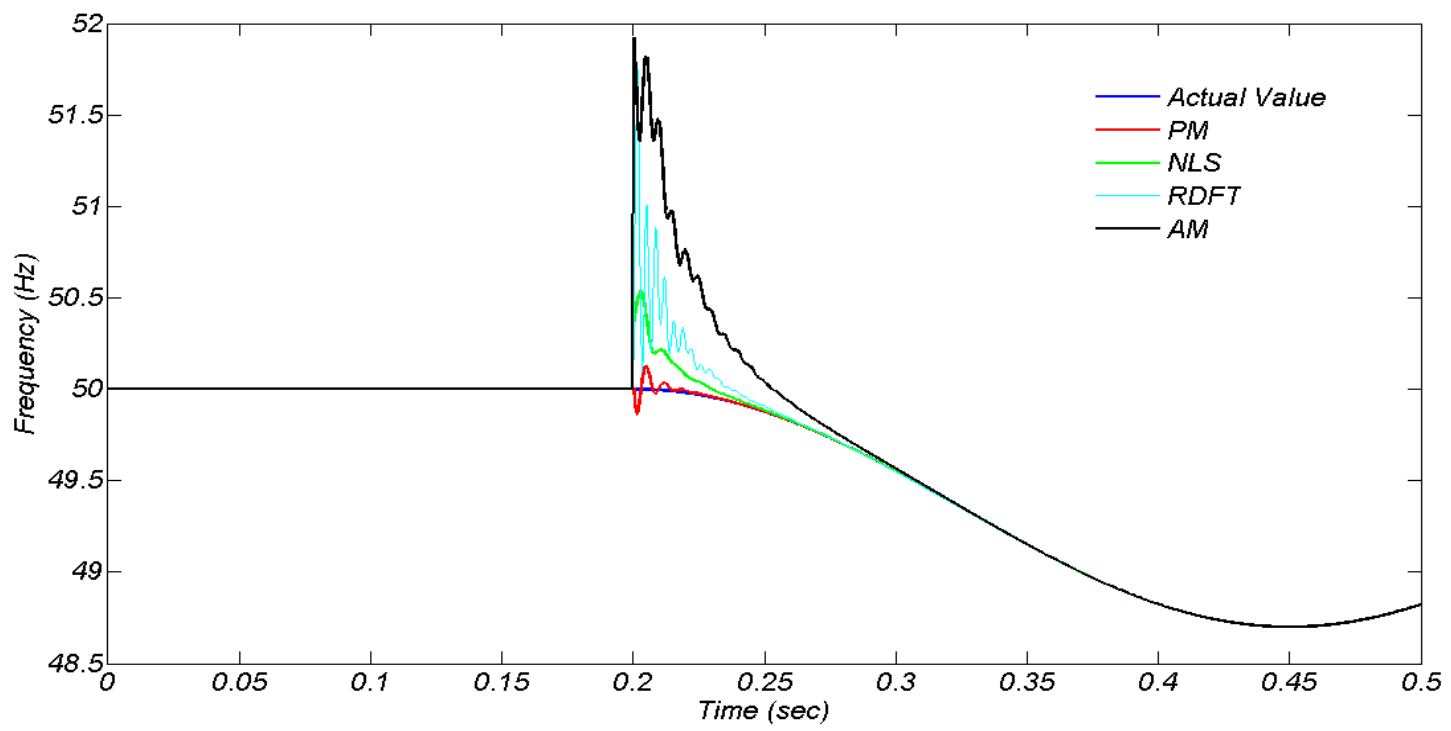

Figure 10. Impact on Frequency during first cycles after fault occurrence

\subsection{Case4}

Figure (11) illustrates the time response of the phase A voltage signal for LG fault condition with a fault incidence angle of $90^{\circ}$ degrees. A magnitude of voltage change of $90 \%(=100 \%-10 \%)$ of the pre-fault amplitude was perceived at this location of the power system selected. as can be seen in figure 11, tension in voltage waveform have been enhanced.

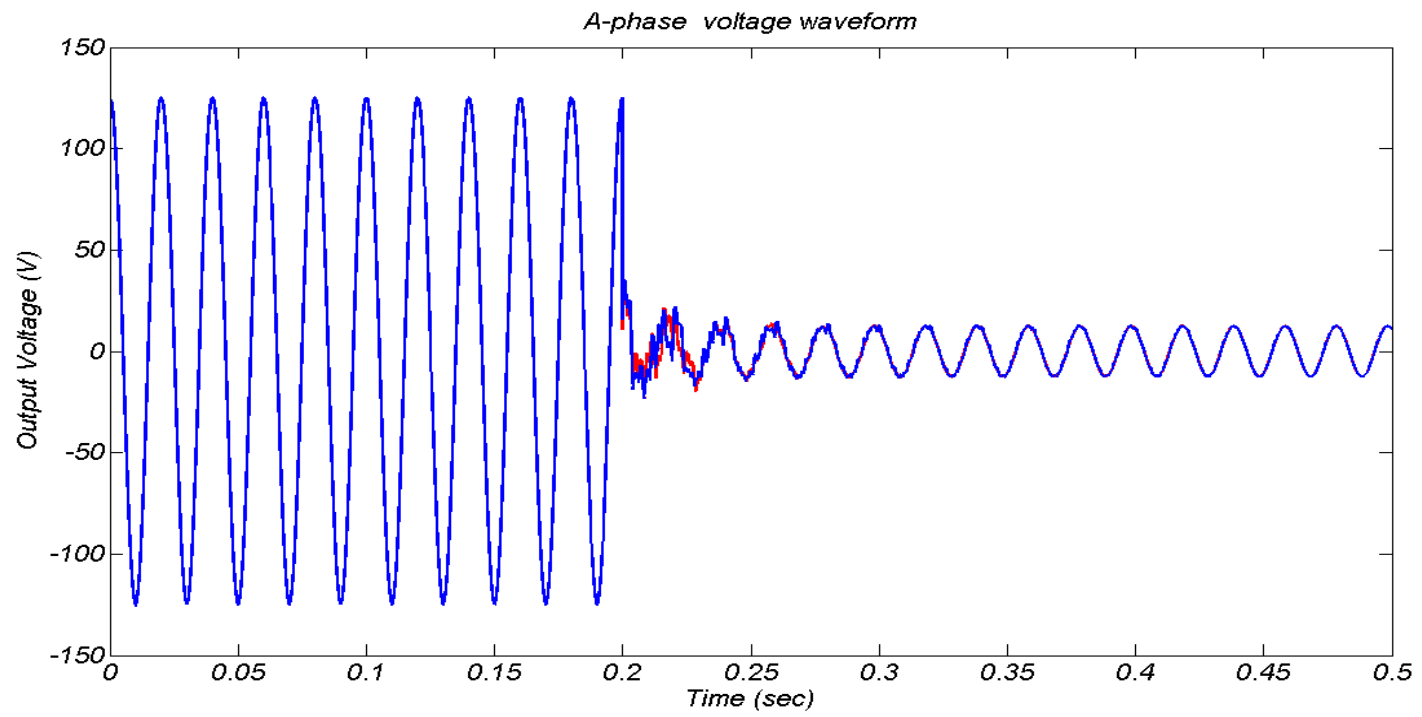

Figure 11. A-phase voltage waveform of the low resistance LG fault

Figure (12) illustrates the frequency of the voltage phasor estimated using proposed approach. As shown in figure 12, proposed approach causes estimated frequency converge to original value after 1 cycle. Despite of tention increment, the proposed approach has more significant accuracy in comparison with the other methods. 


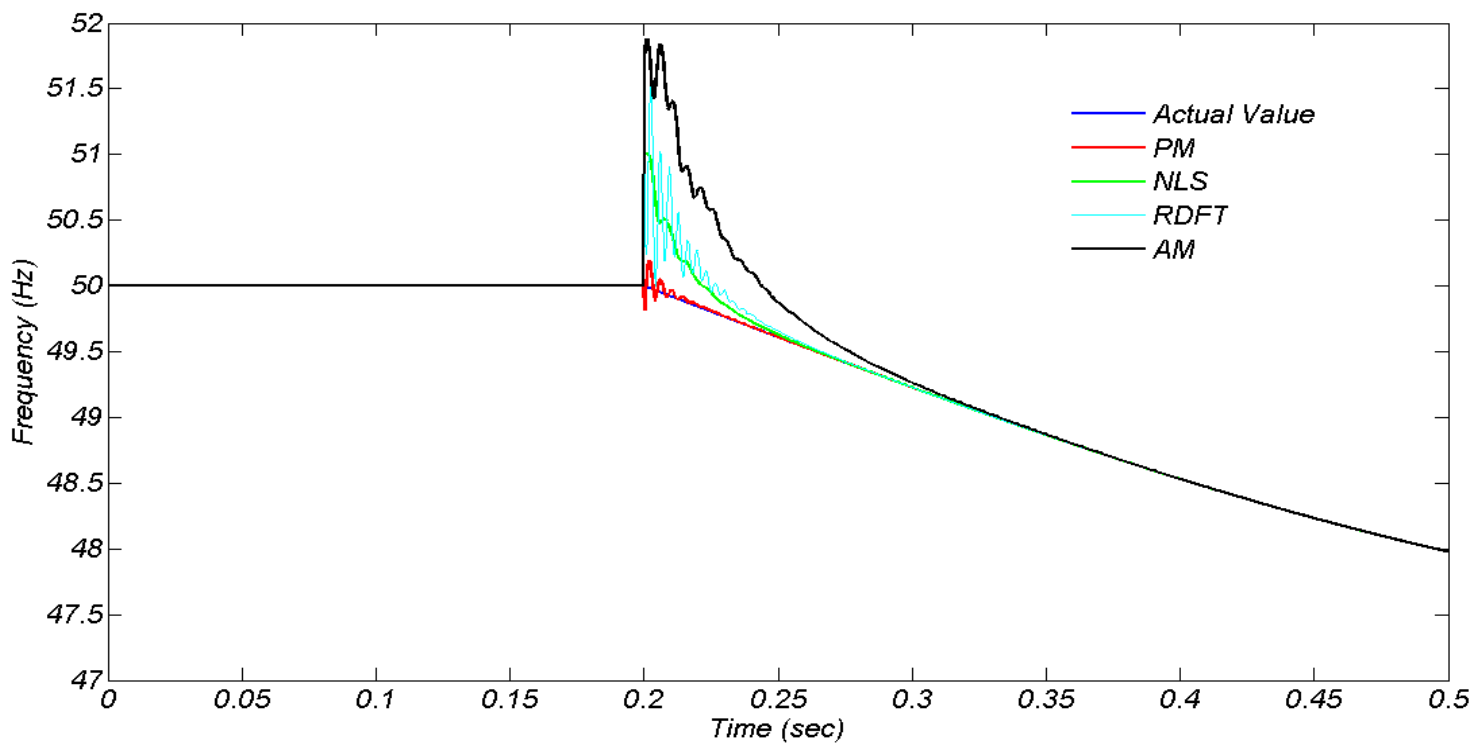

Figure 12. Impact on Frequency during first cycles after fault occurrence

Unlike conventional methods, proposed method has ability to remove transeint component created by CCVT. The CCVT transient components have significant affect to the result of the present methods which causes more instability in result. According to comprehensive fault simulations under numerous fault conditions, the proposed method was practical in terms of the numerical relay design. All of the abovementioned results obviously demonstrate that the proposed method can precisely estimate frequency. Additionally, the proposed method has faster convergence and more precision in comparison with the existent frequency estimation methods.

\section{CONCLUSION}

The impact of transient behavior of CCVT on estimation of the fundamental voltage frequency, which is more important to some of the numerical protective relays, was described in this paper. The transient behavior of instrument measurements causes incorrect estimation within the first few cycles after a fault occurrence have been neglected in fundamental frequency estimation methods. Therefore, the protective system operates incorrectly. The proposed Frequency estimation algorithm was described, developed, demonstrated by a method which is based on Least square error. In order to increase phasor and frequency estimation accuracy, transient behavior of the CCVT, including knowledge of design and the connected burden was applied in Frequency estimation algorithm. This improved the frequency estimation. In order to validate of proposed method many dynamic tests were studied in PSCAD/EMTDC and method performances are shown in simulation results. The results show considerable improvements in precision and also speed convergence. Accordingly, the proposed method is very effective for fundamental frequency estimation of power system signals.

\section{REFERENCES}

[1] M.M. Begovic, P.M. Djuric, S. Dunlap, A.G. Phadke. "Frequency tracking in power networks of harmonics". International Conference on Harmonics in Power Systems. pp. 151-157, 1992.

[2] D.W.P. Thomas, M.S. Woolfson. "Evaluation of frequency tracking methods". IEEE Trans. Power Delivery. Vol. 16, No. 3, pp. 367-371, 2001.

[3] A. Routray, A.K. Pradhan, K.P. Rao. "A novel Kalman filter for frequency estimation of distorted signals in power systems”. IEEE Trans. Instrumentation and Measurement. Vol. 51, No. 3, pp. 469-479, 2002.

[4] P.K. Dash, A.K. Pardhan, G. Panda. "Frequency estimation of distorted power system signals using extended complex Kalman filter”. IEEE Trans. Power Delivery. Vol. 14, No. 3, pp. 761-766, 1999.

[5] V. Kaura, V. Blasko. "Operation of a phase locked loop system under distorted utility conditions". IEEE Trans. Industry Applications. Vol. 33, No. 1, pp. 58-63, 1997.

[6] S.K. Chung. "A phase tracking system for three phase utility interface inverters". IEEE Trans. Power Electronics. Vol. 15, No. 3, pp. 431-438, 2000. 
[7] H. Karimi, M. Karimi-Ghartemani, M.R. Iravani. "Estimation of frequency and its rate of change for applications in power systems”. IEEE Trans. Power Delivery. Vol. 19, No. 2, pp. 472-480, 2004.

[8] M.M. Giray, M.S. Sachdev. "Off-nominal frequency measurement in electric power systems". IEEE Trans. Power Delivery. Vol. 4, No. 3, pp. 1573-1578, 1989.

[9] R. Chudamani, Krishna Vasudevan, C.S. Ramalingam. "Real-Time Estimation of Power System Frequency Using Nonlinear Least Squares”. IEEE Trans. Power Delivery. Vol. 24, No. 3, pp. 1021-1028, 2009.

[10] A.G. Phadke, J.S. Thorp, M.G. Adamiak. "A new measurement technique for tracking voltage phasors, local system frequency and rate of change of frequency”. IEEE Trans. Power Apparatus and Systems. Vol. PAS-102, No.5, pp. 1025-1038, 1983

[11] P.J. Moore, R.D. Carranza, A.T. Johns. "A new numeric technique for high-speed evaluation of power system frequency”. IEE Proceedings - Generation Transmission and Distribution. Vol. 141, No. 5, pp. 529-536, 1994.

[12] J.Z. Yang, C.W. Liu. “A precise calculation of power system frequency”. IEEE Trans. Power Delivery. Vol. 16, No. 3, pp. 361-366, 2001

[13] V.V. Terzija, M.B. Djuric, B.D. Kovacevic. "Voltage phasor and local system frequency estimation using Newtontype algorithms”. IEEE Trans. Power Delivery. Vol. 9, No. 3, pp. 1368-1374, 1994. 0246810

[14] M.D. Kusljevic. “A simple recursive Algorithm for simultaneous magnitude and frequency estimation”. IEEE Trans. Instrumentation and Measurement. Vol. 57, No.6, pp. 1207-1214, 2008.

[15] P.K. Dash, D.P. Swain, A. Routray, A.C. Liew. "An adaptive neural network approach for the estimation of power system frequency”. Electric Power Systems Research. Vol.41, No. 3, pp. 203-210, 1997.

[16] L.L. Lai, W.L. Chan, C.T. Tse, A.T.P. So. "Realtime frequency and harmonic evaluation using artificial neural networks”. IEEE Trans. Power Delivery. Vol. 14, No. 1, pp. 52-59, 1999.

[17] T. Lobos, J. Rezmer. "Real-time determination of power system frequency". IEEE Trans. Instrumentation and Measurement. Vol. 46, No. 4, pp. 877-881, 1997.

[18] Soon-Ryul Nam, Dong-Gyu Lee, Sang-Hee Kang, Seon-Ju Ahn, Joon-Ho Choi. "Fundamental Frequency Estimation in Power Systems Using Complex Prony Analysis”. Journal of Electrical Engineering \& Technology. Vol. 6, No.2, pp. 154-160, 2011.

[19] E. Pajuelo, G. Ramakrishna, M.S. Sachdev. Phasor estimation technique to reduce the impact of coupling capacitor voltage transformer transients. IET Generat. Trans. Distribut. 2 (4) (2008) 588-599.

[20] Working Group of the Relay Input Sources Subcommittee of the Power System Relay Committee. "Transient Response of Coupling Capacitor Voltage Transformers”. IEEE Trans. Power Appl. Syst. vol. PAS-100, no. 12, pp.4811-4814, Dec. 1981.

[21] E. Pajuelo, G. Ramakrishna, M.S. Sachdev. "An Improved Voltage Phasor Estimation Technique to Minimize the Impact of CCVT Transients in Distance Protection". in Proc. Canadian Conference on Electrical and Computer Engineering, 1-4 May 2005, pp. 454-457.

[22] D. Hou, J. Roberts. "Capacitive Voltage Transformers: Transient Overreach Concerns and Solutions for Distance Relaying". in Proc. Canadian Conference on Electrical and Computer Engineering. 26-29 May 1996, pp. 119-125.

[23] D. Angell, D. Hou. "Input Source Error Concerns for Protective Relays". in proc. 60th Annual Conference for Protective Relay Engineers, College Station, TX, 27-29 March 2007, pp. 63-70.

[24] J. Izykowski, B. Kasztenny, E. Rosolowski, M.M. Saha, B. Hillstrom. "Dynamic Compensation of Capacitive Voltage Transformers”. IEEE Trans. Power Delivery. vol. 13, no. 1, pp. 116-122, Jan 1998.

[25] J.R. Lucas, P.G. McLaren, W.W.L. Keerthipala, R.P. Jayasinghe. "Improved Simulation Models for Current and Voltage Transformers in Relay Studies”. IEEE Trans. Power Delivery. vol. 7, no. 1, pp. 152-159, Jan 1992.

[26] Working Group C-5 of the Systems Protection Subcommittee of the IEEE Power System Relaying Committee. "Mathematical Models for Current, Voltage, and Coupling Capacitor Voltage Transformers". IEEE Trans. Power Delivery. vol. 15, no. 1, pp. 62-72, Jan 2000.

[27] J.L. Blackburn. Symmetrical Components for Power Systems Engineering. New York: Marcel Dekker. 1993, p. 427.J. 